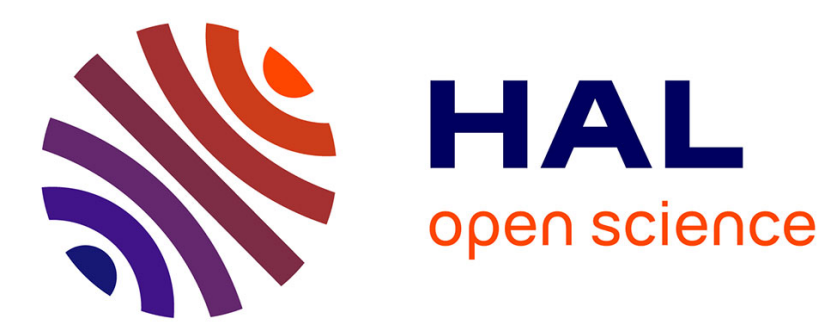

\title{
Scalability of FETI-2LM methods on HPC clusters for Antenna RCS applications
}

\author{
A. Barka, F.X. Roux
}

\section{To cite this version:}

A. Barka, F.X. Roux. Scalability of FETI-2LM methods on HPC clusters for Antenna RCS applications. International Symposium on Antennas and Propagation (ISAP 2014), Dec 2014, KAOSHUONG, Taiwan. hal-01098020

\section{HAL Id: hal-01098020 https://hal.science/hal-01098020}

Submitted on 22 Dec 2014

HAL is a multi-disciplinary open access archive for the deposit and dissemination of scientific research documents, whether they are published or not. The documents may come from teaching and research institutions in France or abroad, or from public or private research centers.
L'archive ouverte pluridisciplinaire HAL, est destinée au dépôt et à la diffusion de documents scientifiques de niveau recherche, publiés ou non, émanant des établissements d'enseignement et de recherche français ou étrangers, des laboratoires publics ou privés. 


\title{
Scalability of FETI-2LM methods on HPC clusters for Antenna RCS applications
}

\author{
André Barka ${ }^{1}$, François-Xavier Roux ${ }^{1}$ \\ ${ }^{1}$ ONERA, The French Aerospace Lab, BP 4025 - 2 Avenue Edouard Belin - F-31055 Toulouse Cedex 4
}

\begin{abstract}
This paper presents the scalability of finite element tearing and interconnecting (FETI-2LM) methods for solving challenging electromagnetic frequency domain problems encountered in the design of low RCS antenna arrays. The scalability of the FETI-2LM method working on Nehalem X5560 cluster will be presented for antenna mock-up.
\end{abstract}

Index Terms - Antennas, RCS, FEM methods, FETI algorithms.

\section{INTRODUCTION OF FETI-2LM FORMULATION}

Simulating the RCS of large finite array constituted with thin conductors, shorting via, thin resistances and dielectric materials is technically very challenging. Indeed, the computation times as well as the memory requirements exceed those available on the most powerful computers. Recently, a parallel algorithm developed on HPC computers called FETI-2LM has been introduced to solve these challenging problems [1]. To deal with large antenna arrays, the general principle of the FETI-2LM methods is to decompose the global computational domain in nonoverlapping sub-domains in which local solution fields are calculated by solving the finite element system with a direct method [1]. We then impose the tangent field continuity on the interfaces by using Lagrange multiplier. It results in a reduced problem on interfaces and can be solved by an iterative method. The solution of the interface problem is used as a boundary condition for evaluating the field in each sub-domain. We denote $\Omega=\Omega_{1} U \Omega_{2} U \ldots U \Omega_{N}$ a partition of the initial computation domain. In each sub-domain $\Omega_{\mathrm{i}}$, we are calculating the scattered fields $\mathrm{E}_{\mathrm{i}}$ verifying on $\Omega_{\mathrm{i}}$ :

$$
\begin{gathered}
\nabla \times\left(\mu_{r, i}^{-1} \nabla \times E_{i}\right)-k_{0}^{2} \varepsilon_{r, i} E_{i}=k_{0}^{2}\left(\varepsilon_{r, i}-\mu_{r, i}^{-1}\right) E_{i n c} \\
n_{e x t} \times \nabla \times E_{i}+j k_{0} n_{e x t} \times\left(n_{e x t} \times E_{i}\right)=0 \\
n_{i} \times\left(\mu_{r, i}^{-1} \nabla \times E_{i, j}\right)+j k_{0} n_{i} \times\left(n_{i} \times E_{i, j}\right)=\Lambda_{i, j} \\
n_{j} \times\left(\mu_{r, j}^{-1} \nabla \times E_{j, i}\right)+j k_{0} n_{j} \times\left(\vec{n}_{j} \times E_{j, i}\right)=\Lambda_{j, i}
\end{gathered}
$$

The vector $E_{\text {incident }}$ is representing a plane wave electric incident field in the volume $\Omega_{\mathrm{i}} . \Gamma_{\mathrm{ABC}}$ represents the boundary of the volume $\Omega_{\mathrm{i}}$, where the field is verifying ABC absorbing boundary conditions (2). We note $\mathrm{E}_{\mathrm{j}, \mathrm{i}}$ the electric field on the interface of the sub domain $\Omega_{\mathrm{i}}$ adjacent to the sub domain $\Omega_{\mathrm{j}}$. On the interfaces $\Gamma_{\mathrm{ij}}$ separating two sub-domains $\Omega_{\mathrm{i}}$ and $\Omega_{\mathrm{j}}$, we impose Robin type boundary conditions by using 2 Lagrange multipliers $\Lambda_{\mathrm{i}, \mathrm{j}}$ and $\Lambda_{\mathrm{j}, \mathrm{I}}(3,4)$.

\section{OPTIMAL STRATEGY FOR MULTIPLE RIGHT AND SIDE}

FETI-2LM is a domain decomposition method which mixes iterative solution of a problem associated with matching conditions along the interfaces between subdomains and direct solution of local problems. The local matrices are factorized once for all. Each iteration of the interface problem requires the solution via a forward-backward substitution of a local problem with a new right-hand-side in every subdomain. The method is parallelized in the MPI message passing programming environment with one subdomain allocated to one process.

For each process, storing one search direction vector on the interface is completely negligible in comparison with the storage requirement of the factorization of the local matrix. The same remark applies for the comparison between the arithmetic complexity of a dot product on the interface and a forward-backward substitution for the local matrix.

This means that Krylov methods with full reorthogonalization like ORTHODIR are the methods of choice for the interface iterations. In the case of multi-righthand-side problems an optimization strategy based on full reorthogonalization for the interface problem can be implemented for decreasing the solution cost for the successive right-hand-sides.

The first method consists in solving the successive problems one by one, with keeping the successive search direction vectors and using them to accelerate the convergence. This approach is similar to a deflation method. The search direction vectors computed for the previous right-hand-sides are kept in memory as well as their product by the interface operator. For each new right-hand-side, the optimal approximate initial solution is searched in the space spanned by all the stored search direction vectors. This requires just a full orthogonalization of the initial residual vector on the interface. The new search direction vectors built during the new ORTHODIR iterations are made A*A orthogonal to all the previously computed search directions. This method requires that the $\mathrm{A}^{*} \mathrm{~A}$ orthogonality conditions are strictly satisfied, so the orthogonalization procedures are based on the modified Gram-Schmidt method. Thanks to this approach, the number of new iterations needed to solve the problem for the successive right-hand-sides is greatly reduced. The additional computing cost is just due to the orthogonalization with all the previously stored interface search directions. This cost remains small compared with the 
forward-backward substitution of the local problem, even with hundreds of search directions.

An improvement of this strategy consists in solving the successive problems not one by one but by blocks of a few (typically 10-20) at the same time. A block of several search directions is computed at each iteration of the interface problem and all the search directions of the block are used for all the right-hand-sides. The same deflation strategy as described before is used for the successive blocks of righthand-sides. The main advantages of this block strategy are as follows. First, instead of performing one forward-backward substitution per iteration, the block strategy performs several forward-backward substitutions at the same time. Since the forward-backward substitution performance is limited by memory bottleneck, the performance of the local direct solver is greatly improved, especially on multi-core compute nodes. Secondly, the computation of the dot products for the orthogonalization procedure requires global reduction operations performed by all the MPI process. The overhead with a single reduction operation is very high. With the block strategy, several dot products can be computed at once, reducing the global overhead due to message passing thanks to multiple reduction operations.

\section{ANTENNA ARRAY RCS}

The array, investigated and designed in the SAFAS project [2], is obtained by periodization of the unit cell described on the Fig. 2. The leading dimension of the unit cell is $7.07 \mathrm{~mm}$ and its height is $20 \mathrm{~mm}$. The unit cell optimized with an equivalent circuit model [3] contains dielectric layers, thin perfectly conductive surfaces and thin $150 \mathrm{Ohms}$ resistive surfaces.

The RCS of the $22 \times 22$ array is given on Fig. 3 for a plane wave at normal incidence and compared with the RCS of a perfectly conductive surface of same dimensions $\left(15.55 \times 15.55 \mathrm{~cm}^{2}\right)$. The simulated RCS reduction is between $10 \mathrm{~dB}$ and $34 \mathrm{~dB}$. The simulation results were obtained with a mesh of the elementary cell composed of 207,000 edges. Four empty unit cells are introduced around the $22 \times 22$ array to keep the $\mathrm{ABC}$ surface sufficiently far away from the scatterer.

The good scalability of the FETI-2LM method is analyzed by considering arrays with progressively increasing size $(7 \times 7$, $20 \times 20,30 \times 30,35 \times 35$, and $44 \times 44)$. The unit cell was done with 207,000 edges for all of the arrays. The elapse time obtained on an SGI X5560 cluster for a convergence lower than $10^{-3}$ is indicated in Fig. 2 . In the case of the $44 \times 44$ array, the total dimension of internal unknowns is 400 million and the size of the interface problem is 26.9 million. The number of iteration required by the iterative method is 150 .

The efficiency of the block RHS strategy is demonstrated in TABLE I in the case of the $7 \times 7$ grid illuminated by 100 incident waves. We observe a speed up of 8.7 while the required memory is increased by a factor 1.84 .

\section{ACKNOWLEDGMENT}

The authors would like to thank the French Ministry of Defense (DGA), the French National Research Agency (ANR) for their support through the Astrid SAFAS Program and the French GENCI consortium for providing HPC resources.

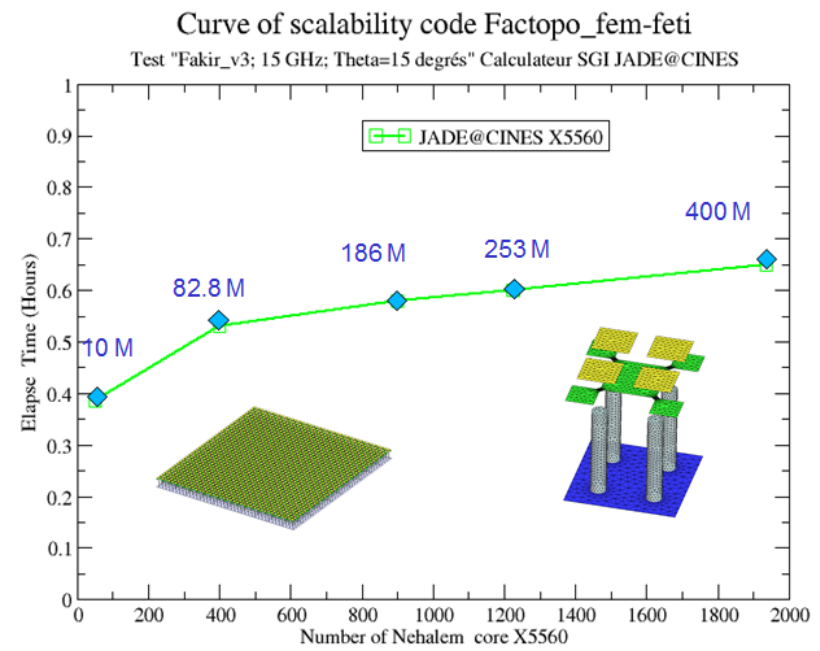

Fig. 2. Geometry and Scalability

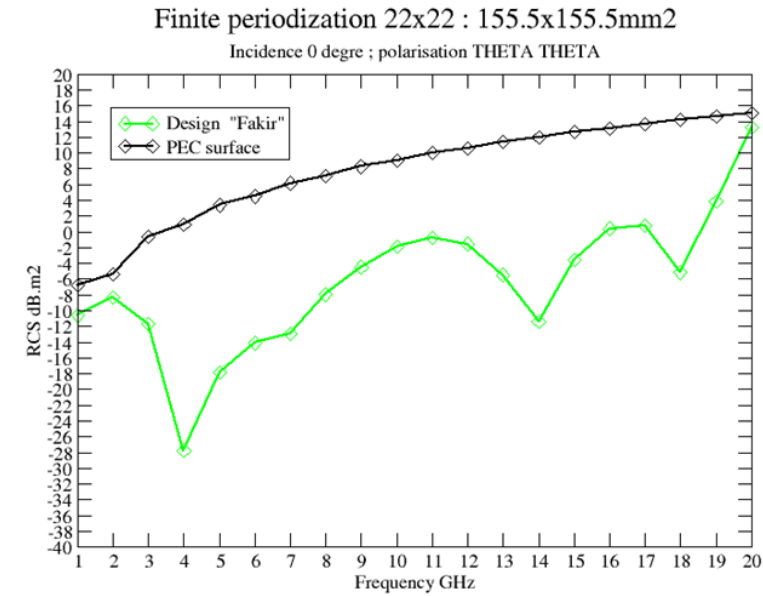

Fig. 3. RCS reduction

TABLE I

PERFORMANCES OF MULTIPLE RHS STRATEGIES ON THE 7X7 GRID

\begin{tabular}{|l|l|l|l|l|l|}
\hline & GRID & RHS & $\begin{array}{l}\text { STORED } \\
\text { DIRECTIONS }\end{array}$ & $\begin{array}{l}\text { ELAPSE } \\
\text { TIME(H) }\end{array}$ & $\begin{array}{l}\text { MEMORY } \\
(\mathrm{GB})\end{array}$ \\
\hline $\begin{array}{l}\text { INITIAL } \\
\text { STRATEGY }\end{array}$ & $7 \times 7$ & 100 & 500 & 6.78 & 0.75 \\
\hline $\begin{array}{l}\text { BLOCK } \\
\text { STRATEGY }\end{array}$ & $7 \times 7$ & 100 & 500 & 0.78 & 1.38 \\
\hline
\end{tabular}

\section{REFERENCES}

[1] F.X Roux, A. Barka, "Chapter 9 on FETI methods", Springer book "Computational Electromagnetics; recent advances and Engineering applications" Raj Mittra Editor, June 2013, DOI 10.1007/978-1-4614$4382-7$

[2] SAFAS project, ANR-12-ASTR-0009-01

[3] S. Varault, A-C Lepage, M. Soiron, X. Begaud "Equivalent circuit model of a self-complementary connected array antenna", Eucap Conference, 6-11 April 2014, La Hague The Netherlands 